\title{
P-0926: Strengthening diabetes and cardiovascular disease surveillance and their risk factors in South Asia
}

\author{
MK Mridha1, JC Chambers2, A Kasturiratne3, JS Kooner2, KI Khawaja4, S Jha5, \\ M Sarkar1, S Ahmed6, A Gupta5, RM Anjana7, W Silva2, P Katulanda8
}

1JPG School of Public Health, BRAC University, Bangladesh, 2Imperial College London, United Kingdom, 3Faculty of Medicine, University of Kelaniya, Sri Lanka, 4Services, Institute of Medical Sciences, Lahore, Pakistan, 5Max Healthcare, New Delhi, India, 6Punjab Institute of Cardiology, Lahore, Pakistan, 7Madras Diabetes Research Foundation, Chennai, India, 8Diabetes Research Unit, University of Colombo, Sri Lanka.

\section{Background}

Accurate knowledge of burden and future trajectories of cardiovascular disease (CVD), Type 2 Diabetes (T2D) and their risk factors is central to formulation of health policy and delivery of effective healthcare interventions for prevention and control. However, the current systems for periodic collection of data on noncommunicable diseases (NCD) are fragmented and incomplete in South Asia.

- Objective: To describe the preliminary findings of the Global Health Research Unit surveillance study which aims to strengthen NCD surveillance systems in Bangladesh, India, Pakistan and Sri Lanka.

\section{Methodology}

- This project is part of the National Institute of Health Research (NIHR) funded Global Health Research Unit on Diabetes and Cardiovascular Diseases in South Asia.

- Consortium includes four countries, India, Pakistan, Sri Lanka and Bangladesh and the project is led by Imperial College London.

- 11 teams with $~ 250$ surveillance sites

- Mobilization of the community and screening (Figure 1)

- 12 stations for each participant covering in 90 minutes (Figure 2)

- Personalised reports
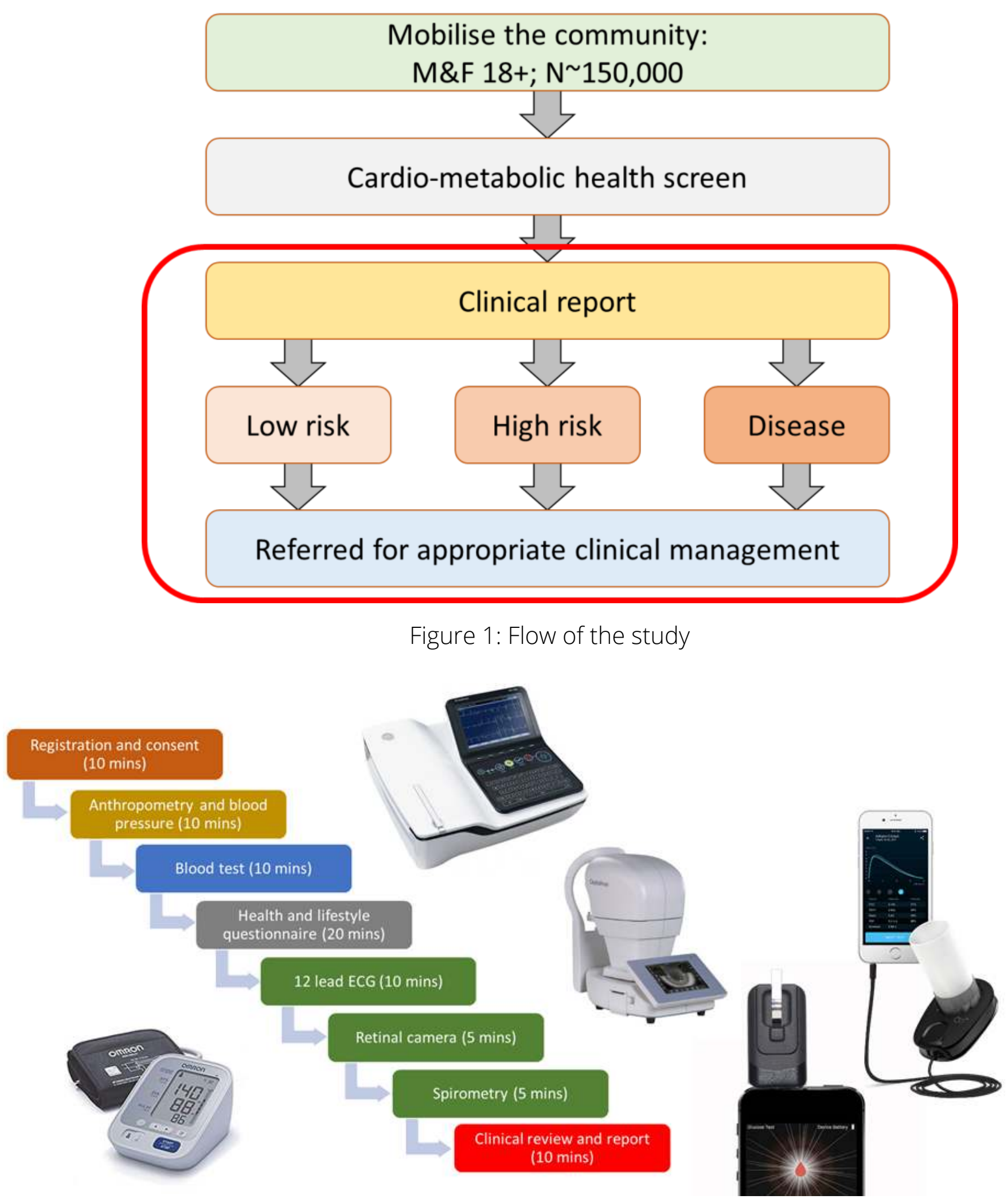

Figure 2: Screening tests with digital data collection

\section{Results}

- So far $\sim 20,000$ participants have been recruited across the four countries

- Distribution of participants in different teams according to the age categories is represented in table 1.

- Comparison of blood pressure in the four countries is shown in figure 3.

- Clinical characteristics of study participants is shown in figure 4.

\begin{tabular}{|l|cc|cc|cc|}
\multicolumn{1}{c|}{} & \multicolumn{8}{c|}{ Age (years) } \\
\cline { 2 - 8 } Teams & \multicolumn{2}{|c|}{$<\mathbf{4 0}$} & \multicolumn{2}{c|}{$\mathbf{4 0 - 6 0}$} & \multicolumn{3}{c|}{$>\mathbf{6 0}$} \\
\hline BRAC_1 & $42.2 \%$ & 299 & $45.8 \%$ & 324 & $12.0 \%$ & $\mathbf{n}$ \\
BRAC_2 & $40.5 \%$ & 251 & $44.8 \%$ & 278 & $14.7 \%$ & 91 \\
DDF_1 & $48.6 \%$ & 277 & $41.4 \%$ & 236 & $10.0 \%$ & 57 \\
DDF_2 & $54.8 \%$ & 241 & $37.0 \%$ & 163 & $8.2 \%$ & 36 \\
MDRF_1 & $23.5 \%$ & 108 & $53.8 \%$ & 247 & $22.7 \%$ & 104 \\
MDRF_2 & $28.0 \%$ & 130 & $53.2 \%$ & 247 & $18.8 \%$ & 87 \\
PIC_Team_1 & $37.2 \%$ & 115 & $48.5 \%$ & 150 & $14.2 \%$ & 44 \\
SIMS_PK & $41.3 \%$ & 198 & $48.2 \%$ & 231 & $10.4 \%$ & 50 \\
UOCteam & $23.3 \%$ & 162 & $51.0 \%$ & 355 & $25.7 \%$ & 179 \\
UOK_1 & $27.6 \%$ & 113 & $48.8 \%$ & 200 & $23.7 \%$ & 97 \\
\hline Total & $\mathbf{3 6 . 7 \%}$ & $\mathbf{1 8 9 4}$ & $\mathbf{4 7 . 2 \%}$ & $\mathbf{2 4 3 1}$ & $\mathbf{1 6 . 1 \%}$ & $\mathbf{8 3 0}$ \\
\hline
\end{tabular}

Table 1: Distribution of participants in age categories

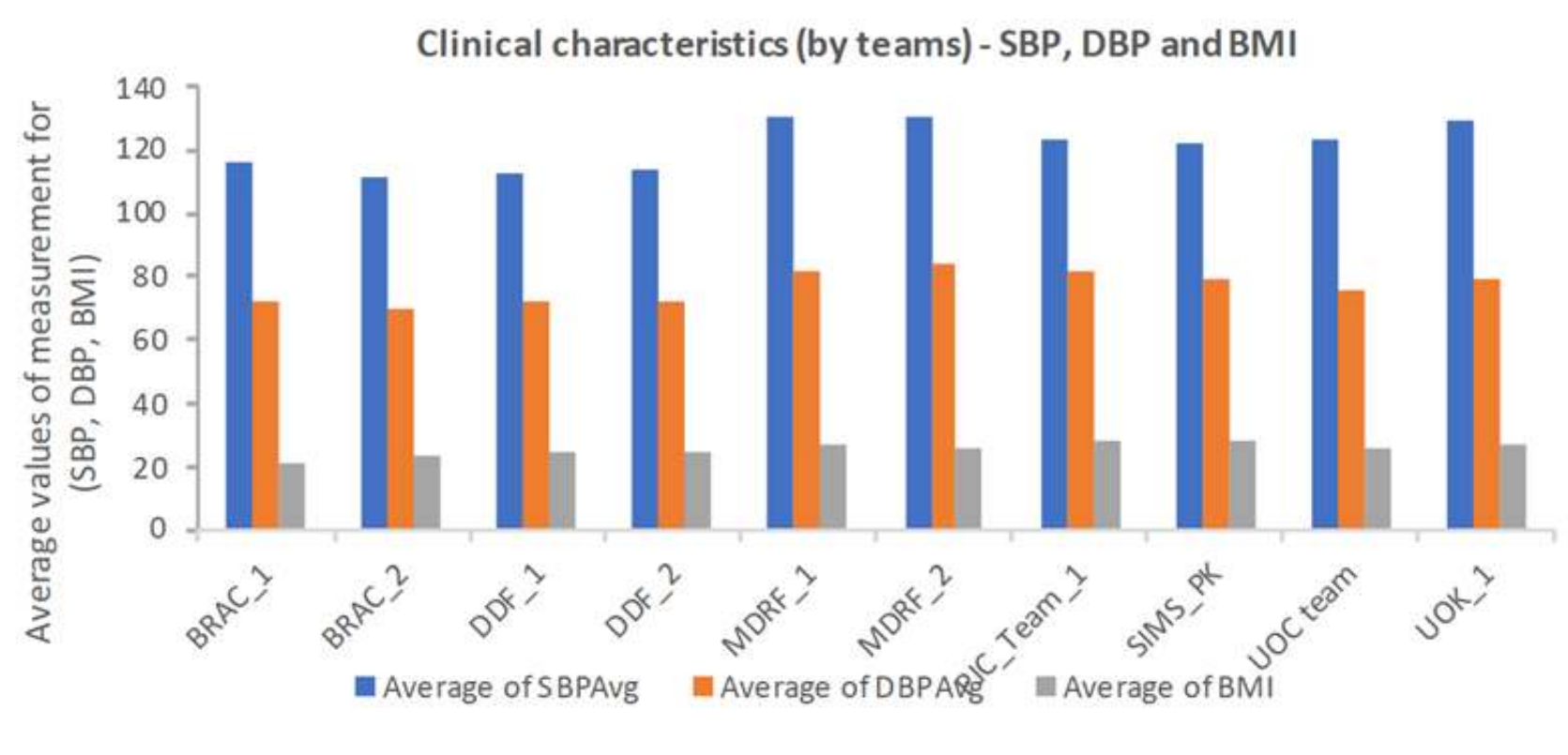

Figure 3: Comparison of Blood Pressure (BP) in countries

\begin{tabular}{|c|c|c|c|c|c|c|}
\hline \multirow{3}{*}{ Teams } & \multicolumn{6}{|c|}{ Clinical characteristic (Categorical Variables) } \\
\hline & \multicolumn{2}{|c|}{ Hypertension status (YES) } & \multicolumn{2}{|c|}{ Diabetes status (YES) } & \multicolumn{2}{|c|}{ Current smokers (YES) } \\
\hline & $\mathbf{n}$ & $\%$ & $\mathbf{n}$ & $\%$ & $\mathbf{n}$ & $\%$ \\
\hline BRAC_1 & 110 & $17.80 \%$ & 19 & $3.07 \%$ & 161 & $26.05 \%$ \\
\hline BRAC_2 & 83 & $15.96 \%$ & 26 & $\square 5.00 \%$ & 123 & $23.65 \%$ \\
\hline DDF_1 & 118 & $20.56 \%$ & 66 & $11.50 \%$ & 79 & $13.76 \%$ \\
\hline DDF_2 & 72 & $16.18 \%$ & 46 & $10.34 \%$ & 46 & $10.34 \%$ \\
\hline MDRF_1 & 72 & $17.27 \%$ & 79 & $18.94 \%$ & 18 & $\square 4.32 \%$ \\
\hline MDRF_2 & 71 & $15.78 \%$ & 92 & $20.44 \%$ & 17 & $3.78 \%$ \\
\hline PIC_Team_1 & 102 & $35.29 \%$ & 37 & $12.80 \%$ & 14 & $\square 4.84 \%$ \\
\hline SIMS_PK & 121 & $30.40 \%$ & 44 & $11.06 \%$ & 32 & $8.04 \%$ \\
\hline UOC team & 174 & $26.77 \%$ & 139 & $21.38 \%$ & 39 & $\square 6.00 \%$ \\
\hline UOK_1 & 80 & $21.74 \%$ & 70 & $19.02 \%$ & 17 & $\square 4.62 \%$ \\
\hline Total & 1003 & $21.21 \%$ & 618 & $13.07 \%$ & 546 & $11.55 \%$ \\
\hline
\end{tabular}

Figure 4: Clinical characteristics of the study population

\section{Conclusion}

This surveillance will continue until 2020 to screen $\sim 1,50,000$ South Asians. Our findings will strengthen NCD surveillance systems in these countries and facilitate transformation of health systems in South Asia through data-driven actions to improve efficiency, effectiveness and quality of healthcare. 